\title{
An Update on College and University Programs in Air Pollution Control
}

\author{
Harold M. Cota \\ California Polytechnic State University
}

\begin{abstract}
A survey of academic programs in air pollution control was made. Results from the 127 schools reporting are tabulated by state. Faculty involved in air pollution instruction are identified. Some conclusions and recommendations are presented.
\end{abstract}

This paper outlines the results of a recent survey which attempted to document who is carrying out academic air pollution control training. A questionnaire directed at faculty associated with these programs was published in the June 1981 issue of JAPCA. This was followed by letters to all non-responding schools who had reported programs in previous studies. ${ }^{2}$ A letter with an abbreviated set of questions was sent as a follow-up to these initial requests for information.

Of the 127 schools responding, 62 answered the detailed questionnaire. A breakdown of the 127 schools indicates: 104 four-year or graduate programs, 12 community college programs, and 10 programs outside the U.S. The complete listing of schools is given in Appendix I. Most of the major air pollution programs available in the U.S. are included.

\section{University Programs}

Air pollution training is an important part of modern engineering education. This is demonstrated in Table I by the wide range of departments in which the training is centered. Other departments reporting training activities not indi-

Dr. Cota is a professor in Environmental Engineering at the California Polytechnic State University, San Luis Obispo, CA 93407, and serves on APCA's Education and Training Committee. cated in the table are identified in Appendix $\mathrm{I}$.

Over $50 \%$ of the universities responding indicated their programs were accredited by ABET. Several indicated accreditation by other professional organizations. Faculty involved with air pollution training at the time the survey was completed are listed in Appendix I. An average of 4 faculty members per established program was found.

Table I. Academic departments responsible for air pollution control training.

\begin{tabular}{lr}
\hline Civil Engineering & 37 \\
Chemical Engineering & 21 \\
Environmental Engineering & 17 \\
Environmental Science & 15 \\
Mechanical Engineering & 11 \\
Environmental Health Science & 7 \\
Meteorology & 3 \\
Chemistry & 2 \\
\hline
\end{tabular}

\section{Curriculum}

The typical curriculum pattern remains one introductory course in air pollution at the undergraduate or graduate level. The schools offering more extensive air pollution training programs are limited. Figure 1 and Table II summarize these results. The more specialized courses are usually of fered at the graduate level. Schools of fering more extensive air pollution training programs are limited in number.

Water pollution control course work is also an integral part of the curriculum

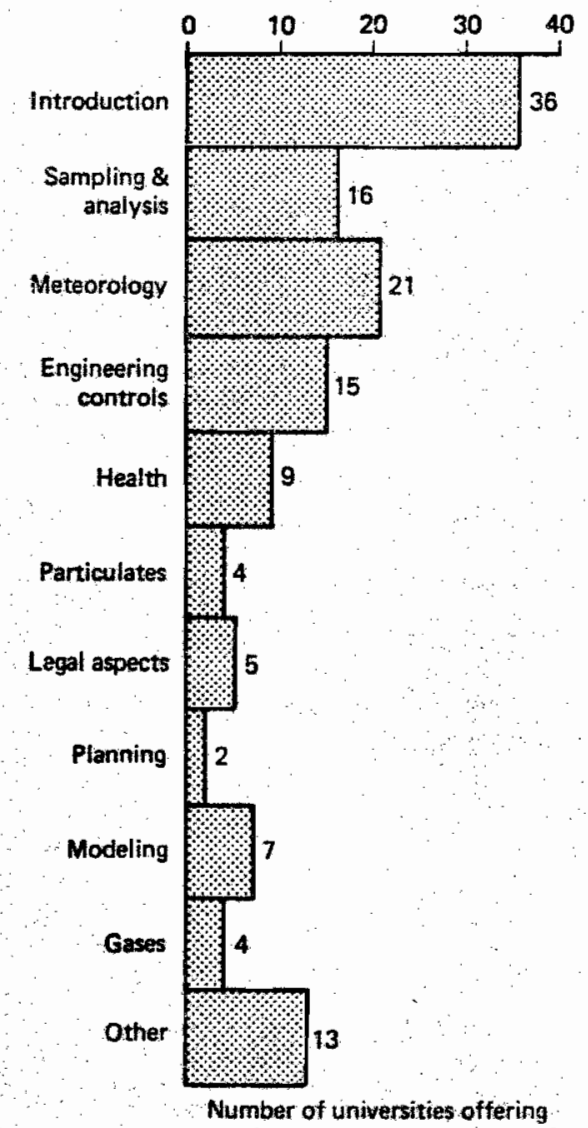

Figure 1. Air pollution coorse work avalable 162 universities responding).

of the schools surveyed. Other related specialized course work available included solid waste management, water resources, radiation and noise. This is

Cepyriphtit 1983-Air Pollution Contral Aasaciation 
Table 11. Number of schools offering related courses (127 universities reporting).

\begin{tabular}{lr} 
Water Pollution & 101 \\
Solid Waste Management & 31 \\
Water Resources & 52 \\
Radiation & 19 \\
Noise & 21 \\
Other & 25 \\
\hline
\end{tabular}

illustrated in Table II. In addition to the typical academic course work, 18 universities are involved in giving short courses in air pollution control.

\section{Students}

Enrollment of students in programs that qualified them to work in air pollution control are shown in Table III. Students graduating in these programs in 1980 included 398 at the B.S. level, 222 at the M.S. level, and 24 with Ph.D.

Faculty were asked to estimate where their students who work in some area of air pollution control find employment. Results were averaged and are summarized in Table IV. This represented the employment picture in 1981.

\section{Financial Support}

Universities were asked to evaluate their air pollution training efforts between 1981 and 1984. More than half $(33 / 62)$ expected their program to re-

Table III. Number of students enrolled qualified to work in air pollution control (62 universities reporting).

\begin{tabular}{lrr}
\hline Degree & 1980 & 1981 \\
\hline B.S. & 1105 & 1254 \\
M.S. & 576 & 518 \\
Ph.D. & 96 & 116 \\
\hline
\end{tabular}

main unchanged, 10 expected their program to become larger, 8 expected their program to get smaller, while the remaining 11 predicted only some change.

Table IV. Percent employment by sector.

\begin{tabular}{lr}
\hline \multicolumn{1}{c}{ Sector } & $\%$ \\
\hline Industry (pollution source) & 34 \\
Control equipment & 3 \\
Instrument manufacture & 1 \\
Large consulting firm & 14 \\
Small consulting firm & 6 \\
Local agency & 8 \\
State agency & 14 \\
Federal agency & 12 \\
University & 6 \\
Other & 3 \\
\hline
\end{tabular}

Financial support is vital for any academic program. This is especially true at the graduate level. It was interesting to determine where the support will need to come from to maintain programs at the current size. Table V summarizes the results.

\section{Conclusions}

The commitment of universities around the U.S. and in other countries continues with respect to air pollution control. The challenge to achieve acceptable environmental conditions with economic growth requires universities to offer air pollution training to both the specialist and the nonspecialist. To effectively carry this out requires both students and experienced faculty.

Students in all disciplines need to be reminded how their professional decisions affect the environment. Several schools use an introductory air pollution course this way. Students need to be encouraged by faculty in their major department to take the appropriate courses. Student chapters, national paper competitions, APCA Section and Chapter recognitions and scholarships will help motivate outstanding students in all disciplines to take coursework required to understand air pollution control.

In order for faculty to be able to effectively teach both the general and specialized courses, they must have the opportunity for professional development. Too often this is not considered adequately in the schedules and budgets of universities. Consulting and research are certainly important, but so is participation in professional societies like APCA. Funds for continuing professional development, as well as facilities, are vital and should be considered by industry and government as part of their stake in the future. The reader is encouraged to contact one of the air pollution programs reported in this survey to learn more about it and its special needs.

Recognizing the incompleteness and the uncertainties that exist in the present study, it is believed that the basic data reported should be useful. Additions, corrections, and comments will be appreciated.

Table V. Source of funds required to continue programs

\begin{tabular}{lr}
\hline \multicolumn{1}{c}{ Source } & $\%$ \\
\hline State & 58 \\
Federal & 29 \\
Research grants & 45 \\
Industry & 16 \\
University & 8 \\
Pays for itself & 11 \\
\hline
\end{tabular}

\section{Reference}

1. A. 'T'. Rossano, H. M. Cota, "University programs in air pollution control: review and outlook," JAPCA 28: 1106 (1978).

Appendix I. Colleges offering air pollution coursework in the U.S. and Canada-1982.

\begin{tabular}{|c|c|c|c|c|c|c|}
\hline State & College or University & $\begin{array}{c}\text { No. of } \\
\text { Air } \\
\text { Pollution } \\
\text { Courses }^{a}\end{array}$ & $\begin{array}{l}\text { No. of } \\
\text { Water } \\
\text { Pollution } \\
\text { Courses }^{\text {a }}\end{array}$ & Program In & Degrees & Name \\
\hline ALABAMA & U. of Alabama & $1(1)$ & $7(4)$ & $\mathrm{CE}$ & $\mathrm{MS}, \mathrm{PhD}$ & G. P. Whittle \\
\hline ALASKA & U. of Alaska & 1 & 2 & Env Q Engr & MS & Oscar Dickason \\
\hline ARIZONA & Arizona State U. & 1 & $8(5)$ & $\mathrm{CE}$ & & J. W. KJock, T. E. Higgins \\
\hline \multirow[t]{4}{*}{ CALIFORNIA } & Calif. Inst. of Technology & 5 & 17 & EnvE Sci & BS, MS, PhD & R. C. Flagan \\
\hline & Cal. State U.-Sacramento & $1(1)$ & $2(3)$ & $\mathrm{CE}$ & $\mathrm{BS}, \mathrm{MS}$ & A. Ranzieri, K. Kerri \\
\hline & Cal. State U.-San Jose & $2(3)$ & & Met & $\mathrm{BS}, \mathrm{MS}$ & R. Bornstein, K. MacKay \\
\hline & $\begin{array}{l}\text { Cal. Poly. State U.-San Luis } \\
\text { Obispo }\end{array}$ & (7) & (5) & CE, EnvE, ME & $\mathrm{BS}, \mathrm{ME}$ & $\begin{array}{l}\text { H. M. Cota, R. G. Keif, D. M. } \\
\text { Misic, W. E. Holtz, W. E. } \\
\text { Clark }\end{array}$ \\
\hline$\because$ & Humboldt State U. & (2) & (4) & EnvRE & $\mathrm{BS}, \mathrm{MS}$ & C. M. Anderson \\
\hline & Stanford University & $1(1)$ & 0 & ME & BS, MS, PhD & C. H. Kruger \\
\hline & U. of Calif.-Berkeley & $10(2)$ & $15(4)$ & $\begin{array}{l}\text { PH, ChE, CE, } \\
\text { ME, Ch }\end{array}$ & MS, PhD & D. H. Thomas \\
\hline & U. of Calif.-Davis & $2(9)$ & $13(9)$ & $\mathrm{CE}$ & $\mathrm{BS}, \mathrm{MS}, \mathrm{PhD}$ & D. P.Chang \\
\hline & U. of Calif,-Irvine & $3(3)$ & $4(3)$ & $M E$ & BS, MS, PhD & G. Samuelson \\
\hline & U. of Calif.-Los Angeles & $2(4)$ & $3(3)$ & Ap Sci, Engr & $\mathrm{BS}, \mathrm{MS}, \mathrm{PhD}$ & R. I. Perrine \\
\hline & U. of Calif.-Riverside & (6) & (8) & EnvSci & $\mathrm{AB}, \mathrm{MS}$ & P. H. Diage \\
\hline
\end{tabular}




\begin{tabular}{|c|c|c|c|c|c|c|}
\hline State & College or University & $\begin{array}{l}\text { No. of } \\
\text { Air } \\
\text { Pollution } \\
\text { Courses }^{*}\end{array}$ & $\begin{array}{l}\text { No. of } \\
\text { Water } \\
\text { Pollution } \\
\text { Courses }\end{array}$ & Program In & Degrees & Name \\
\hline COLORADO & Colorado State U. & 5 & $7(5)$ & EE, EnvE, ME & $\mathrm{BS}, \mathrm{MS}, \mathrm{PhD}$ & $\begin{array}{l}\text { R. Pearson, Atm Sci; E. Reiter, } \\
\text { Atm Sci; D. Hendricks, P. } \\
\text { Sinclair, Atm Sci; C. Turner, } \\
\text { H. Edwards, ME; T. Sanders }\end{array}$ \\
\hline CONNECTICUT & U: of Hartford & (1) & (8) & $\mathrm{CE}$ & BS & R. Dalphin, C. Hemond, ME \\
\hline WASHINGT'ON, DC & Howard University & $3(1)$ & $11(4)$ & $\mathrm{CE}$ & BS, MS & $\begin{array}{l}\text { J. H. Johnson, R. C. Chawla, } \\
\text { ChE; H. Cole, Ecology }\end{array}$ \\
\hline DELAWARE & U. of Delaware & $1(1)$ & $5(3)$ & $\mathrm{CE}, \mathrm{ChE}$ & BS, MS, PhD & L. Spielman \\
\hline FLORIDA & $\begin{array}{l}\text { Florida Int'l. U. } \\
\text { Florida Tech. U. } \\
\text { U. of Central Florida } \\
\text { U. of Florida }\end{array}$ & $\begin{array}{l}4(1) \\
4 \\
3(3) \\
5(1)\end{array}$ & $\begin{array}{l}6(4) \\
6(4) \\
10(7)\end{array}$ & $\begin{array}{l}\text { CE, EnvE } \\
\text { EnvE } \\
\text { CE, EnvS. } \\
\text { EnvSci }\end{array}$ & $\begin{array}{l}\text { BS, MS } \\
\text { BSE, MSE, MS } \\
\text { BSE, MSE, MS } \\
\text { BS, MS, PhD }\end{array}$ & $\begin{array}{l}\text { J. T. Viliate } \\
\text { J. Hartman } \\
\text { Dr. Wanulista, D. Cooper } \\
\text { D. Lundgren, E. Allen }\end{array}$ \\
\hline HAWAII & U. of Hawaii & 3 & (1) & Met & & Wan-Cheng Chiu \\
\hline IDAHO & U. of Idaho & $1(2)$ & & Chem & BS, MS, PhD & $\begin{array}{l}\text { S. Farwell, D. Adams, C. Wai, } \\
\text { D. Brown }\end{array}$ \\
\hline ILLINOIS & $\begin{array}{l}\text { DePaul U. } \\
\text { Governors State U. } \\
\text { Ill. Inst. of Technology } \\
\text { Northwestern U. } \\
\text { So. Illinois U.-Edwardsville }\end{array}$ & $\begin{array}{l}(1) \\
6(11) \\
7(1) \\
1 \\
9(9) \\
\end{array}$ & $\begin{array}{l}(3) \\
3(8) \\
10(2) \\
7(2) \\
2(5)\end{array}$ & $\begin{array}{l}\text { Chem } \\
\text { EnvSci } \\
\text { EnvE } \\
\text { CE } \\
\text { CE, EnvSt }\end{array}$ & $\begin{array}{l}\text { BS } \\
\text { BA, MS } \\
\text { MS, PhD } \\
\text { BS, MS, PhD } \\
\text { BS, MS }\end{array}$ & $\begin{array}{l}\text { F. Breitbeil } \\
\text { E. Cehelnik, H. Sievering } \\
\text { R. Edgar } \\
\text { H. Cember } \\
\text { G. Arnold, P. Kokoropoulos, C. } \\
\text { Thornton, D. McCabe, H. } \\
\text { Kircher, L. McAneny, S. } \\
\text { Hall, D. Myer, D. Rands, R. } \\
\text { Kohn }\end{array}$ \\
\hline 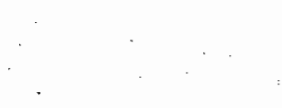 & S. Illinois U._Carbondale & $2(2)$ & $6(2)$ & Th, EnvE & $\mathrm{BS}, \mathrm{MS}$ & $\begin{array}{l}\text { H. Hesketh, T. Squire, W. } \\
\text { O'Brien, S. Rajaa, T. Petires, } \\
\text { J. Chen }\end{array}$ \\
\hline , & $\begin{array}{l}\text { U. of Illinois-Urbana } \\
\text { U. of Illinois-Chicago }\end{array}$ & $\begin{array}{l}8(8) \\
4(13)\end{array}$ & $\begin{array}{l}12 \\
3\end{array}$ & $\begin{array}{l}\mathrm{CE} \\
\text { EnvHealth } \mathrm{Sc}\end{array}$ & $\begin{array}{l}\text { MS, PhD } \\
\text { MPH, MS, } \\
\text { DrPH, PhD }\end{array}$ & $\begin{array}{l}\text { J. Stukel, P. K. Hophe } \\
\text { R. Wadden, R. Allen, L. } \\
\text { Babcock, E. Hermanos, W. } \\
\text { Hallenbeck, J. Holden, W. } \\
\text { Clark, B. Brenniman }\end{array}$ \\
\hline & Midwest College of Engr. & 3 & (3) & $\mathrm{CE}, \mathrm{ChE}$ & $\mathrm{BS}, \mathrm{MS}$ & W. Koch \\
\hline INDIANA & $\begin{array}{l}\text { Purdue University } \\
\text { Rose-Hulman Inst. of } \\
\text { Technology }\end{array}$ & $\begin{array}{l}3(1) \\
0\end{array}$ & $\begin{array}{l}4(2) \\
0\end{array}$ & $\begin{array}{l}\mathrm{CE}, \mathrm{ME} \\
\mathrm{ChE}, \mathrm{CE}\end{array}$ & $\mathrm{BS}, \mathrm{MS}, \mathrm{PhD}$ & $\begin{array}{l}\text { R. B. Jacko, C. Warner } \\
\text { M. Thomas }\end{array}$ \\
\hline $\begin{array}{l}\text { IOWA } \\
\text { KANSAS }\end{array}$ & $\begin{array}{l}\text { U. of Iowa } \\
\text { U. of Kansas }\end{array}$ & $\begin{array}{l}2(2) \\
6(1)\end{array}$ & $\begin{array}{l}14(9) \\
12(5)\end{array}$ & $\begin{array}{l}\text { EnvE, CE } \\
\text { CE }\end{array}$ & $\begin{array}{l}\text { MS, PhD } \\
\text { MS, PhD }\end{array}$ & $\begin{array}{l}\text { R. R. Dague } \\
\text { D. Lane, R. McKinney, C. } \\
\text { Burkhead }\end{array}$ \\
\hline KENTUCKY & U. of Kentucky & 2 & 4 & ChE & $\mathrm{MS}, \mathrm{PhD}$ & $\begin{array}{l}\text { L. Peters, D. Bhattacharyya, R. } \\
\text { Peck-ME } \\
\text { Martin, Fleishman }\end{array}$ \\
\hline " & $\begin{array}{l}\text { U. of Louisville } \\
\text { E. Kentucky U. } \\
\text { W. Kentucky U. }\end{array}$ & (1) & (7) & EnvH Sci & $\begin{array}{l}\text { BS } \\
\text { BS }\end{array}$ & $\begin{array}{l}\text { J. H. McLean } \\
\text { D. Rowe }\end{array}$ \\
\hline LOUISIANA & McNeese State U. & $2(2)$ & $2(5)$ & EnvSci & BS, MS & $\begin{array}{l}\text { V. Monsour, D. Cassorly, L. } \\
\text { Stevenson, G. Fister, S. } \\
\text { Chang }\end{array}$ \\
\hline MARYLAND & Johns Hopkins U. & $21(0)$ & $8(0)$ & EnvH Sci & $\begin{array}{l}\text { MHS, ScM, } \\
\text { ScD, PhD }\end{array}$ & C. Billings \\
\hline & U. of Maryland & $5(3)$ & $3(1)$ & Met & $\mathrm{BS}, \mathrm{MS}, \mathrm{PhD}$ & $\begin{array}{l}\text { berg, J. Gentry, G. } \\
\text { n, W. Zoller }\end{array}$ \\
\hline $\begin{array}{c}\text { MASSACHUSETTS } \\
. \\
\end{array}$ & Harvard U. & 8 & 3 & PH, EnvH Sci & $\mathrm{MS}, \mathrm{ScD}$ & $\begin{array}{l}\text { W. Burgess, D. Cooper, M. } \\
\text { Ellenbecker, M. First, W. } \\
\text { Hinds, D. Leith, D. Moeller, } \\
\text { T. Smith, J. Spencer, D. } \\
\text { Leith }\end{array}$ \\
\hline MICHIGAN & $\begin{array}{l}\text { Ferris State College } \\
\text { Michigan State U. }\end{array}$ & $\begin{array}{l}(2) \\
4(1)\end{array}$ & $\begin{array}{l}(3) \\
6(4)\end{array}$ & $\begin{array}{l}\text { EnvQ } \\
\text { CE, San.E }\end{array}$ & $\begin{array}{l}\text { BS } \\
\text { MS, } \mathrm{PhD}\end{array}$ & $\begin{array}{l}\text { J. Fleming } \\
\text { M. Davis, J. Eastman, R. } \\
\text { Boumeester, D. Wiggers }\end{array}$ \\
\hline . & U. of Michigan & 5 & 2 & PH, EnvE & $\mathrm{MS}, \mathrm{PhD}$ & $\begin{array}{l}\text { T. Armstrong, L. Fine, M. } \\
\text { Hilbert, R. Smith, L. } \\
\text { Whitehead }\end{array}$ \\
\hline & Wayne State U. & 7 & $18(10)$ & ChE, Oc EnvH & MS, PhD & $\begin{array}{l}\text { R. Kummler, R. Mickelson, P. } \\
\text { Wagner, T. L. Singh-ME }\end{array}$ \\
\hline MINNESOTA & U. of Minnesota & 5 & 5 & $\begin{array}{l}\text { EnvEH, ME, } \\
\mathrm{CE}\end{array}$ & $\mathrm{MS}, \mathrm{MPH}, \mathrm{PhD}$ & D. Barber; M. Gallagner \\
\hline MISSOURI & $\begin{array}{l}\text { St. Louis U. } \\
\text { Washington U. } \\
\text { U. of Missouri-Rolla }\end{array}$ & $\begin{array}{l}1(1) \\
2(2) \\
2\end{array}$ & $\begin{array}{l}0 \\
4(2)\end{array}$ & $\begin{array}{l}\text { E. Atm. Sci } \\
\text { ME } \\
\text { CE }\end{array}$ & $\begin{array}{l}\text { BS, MS, PhD } \\
\text { MS, DSc } \\
\text { BS, MS, PhD }\end{array}$ & $\begin{array}{l}\text { J. Moore } \\
\text { R. Husar, W. White } \\
\text { C. Billings, Dr. Parish, K. } \\
\text { Terkonda }\end{array}$ \\
\hline MONTANA & Montana State & $1(1)$ & $11(2)$ & $\mathrm{CE}$ & & $\begin{array}{l}\text { H. Peavy, A. Amirtharajah, W. } \\
\text { Characklis, R. Sauks }\end{array}$ \\
\hline NEBRASKA & U. of Nebraska & 1 . & & ChE & BS, MS & L. C. Tao \\
\hline
\end{tabular}




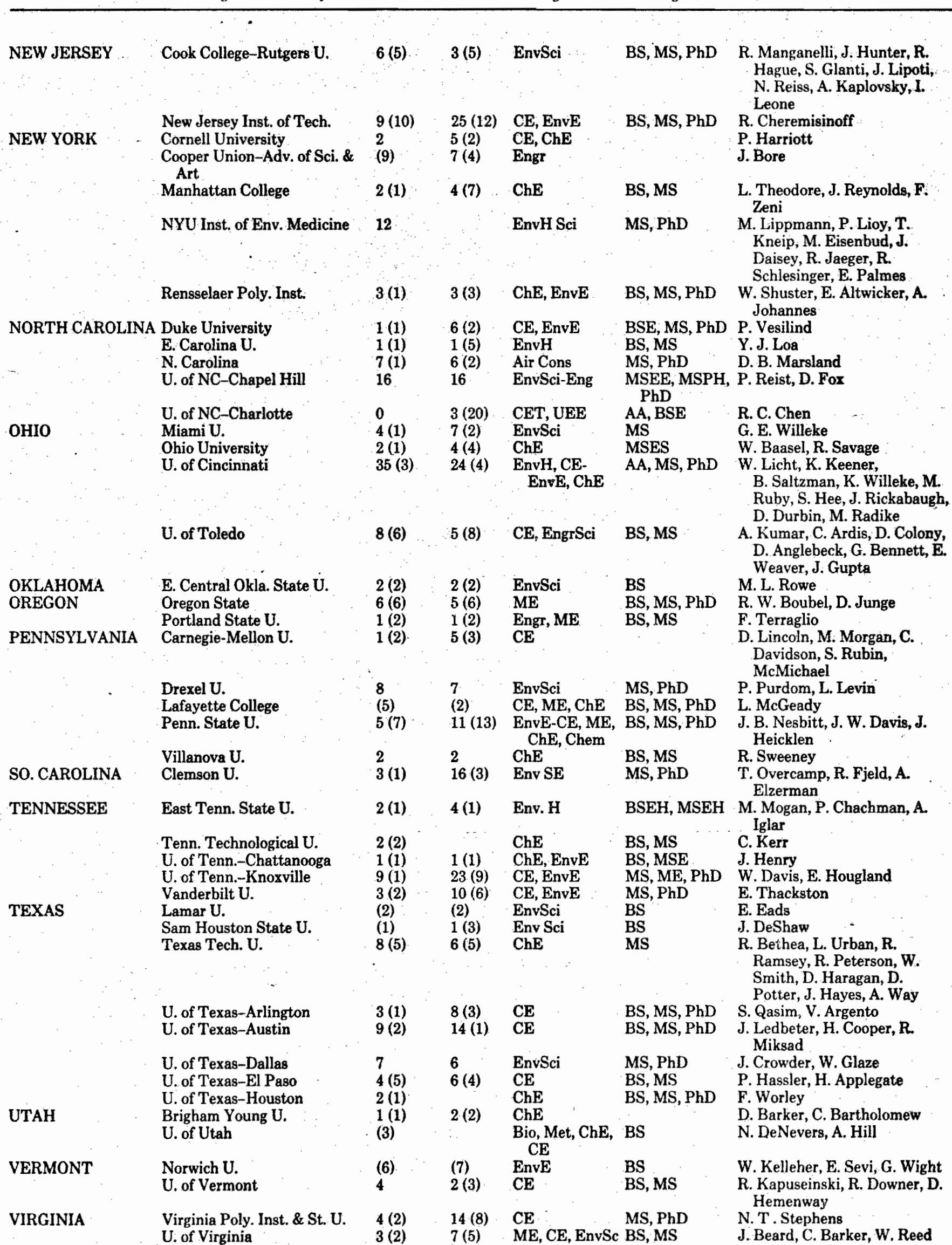




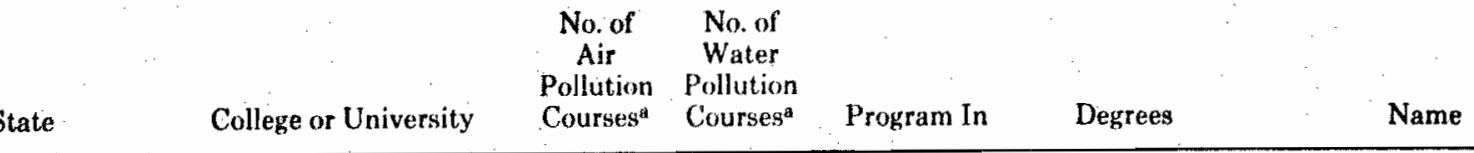

\begin{tabular}{|c|c|c|c|c|c|c|}
\hline WASHINGTON & Washington State U. & $7(3)$ & (7) & ChE & $\mathrm{MS}, \mathrm{PhD}$ & $\begin{array}{l}\text { E. Robinson, D. Cronn, H. } \\
\text { Westberg, R. Koppe, M. } \\
\text { Pack. B. Lamb, M. Cambell, } \\
\text { W. Thomson }\end{array}$ \\
\hline & U. of Washington & $10(3)$ & & $\mathrm{CE}$ & $\begin{array}{l}\text { DSc, BSCE, } \\
\text { MS, PhD }\end{array}$ & M. Pilat \\
\hline WEST VIRGINIA & $\begin{array}{l}\text { W. Virginia Coll. of Grad. } \\
\text { Studies }\end{array}$ & 6 & 5 & ChE, EnvSt & $\mathrm{MS}, \mathrm{ChE}$ & F. Kroesser \\
\hline WISCONSIN & $\begin{array}{l}\text { Marquette U. } \\
\text { U. of Wisconsin-Milwaukee }\end{array}$ & $\begin{array}{l}(2) \\
2(2)\end{array}$ & $\begin{array}{l}9(6) \\
3(3)\end{array}$ & $\begin{array}{l}\mathrm{CE} \\
\mathrm{CE}, \mathrm{ME}\end{array}$ & $\begin{array}{l}\text { MS, BSCE } \\
\text { BS, MS }\end{array}$ & $\begin{array}{l}\text { A. Zanoni } \\
\text { K. Tsao, Miller, Bayer, } \\
\text { Ballentine, Hsu, Skider }\end{array}$ \\
\hline CANADA & Universite de Sherbrooke & $1(1)$ & $2(1)$ & $\mathrm{ChE}$ & BS, MS & $\begin{array}{l}\text { M. Beerh, M. Boulos, J. Jones, } \\
\text { M. deBroissia, E. Chornet }\end{array}$ \\
\hline$\therefore$ & $\begin{array}{l}\text { U. of Calgary } \\
\text { U. of Windsor } \\
\text { U. of Toronto } \\
\text { U. of Sherbrooke }\end{array}$ & $\begin{array}{l}1(1) \\
2(1) \\
8(2) \\
(1)\end{array}$ & $\begin{array}{l}1 \\
4(4) \\
8(2) \\
(1)\end{array}$ & $\begin{array}{l}\text { ChE } \\
\text { HortSci, ChE } \\
\text { CE, Chem, ME } \\
\text { ChE }\end{array}$ & $\begin{array}{l}\text { MSc } \\
\text { BS, MS, PhD } \\
\text { MS, PhD }\end{array}$ & $\begin{array}{l}\text { M. Montari } \\
\text { A. Gnyp, C. St. Pierre } \\
\text { J. G. Henry, J. W. Smith }\end{array}$ \\
\hline SPAIN & Escuela & 3 & 1 & CE & MS & $\begin{array}{l}\text { J. Garcia-Heras, J. Hernando," } \\
\text { J. Frigany }\end{array}$ \\
\hline
\end{tabular}

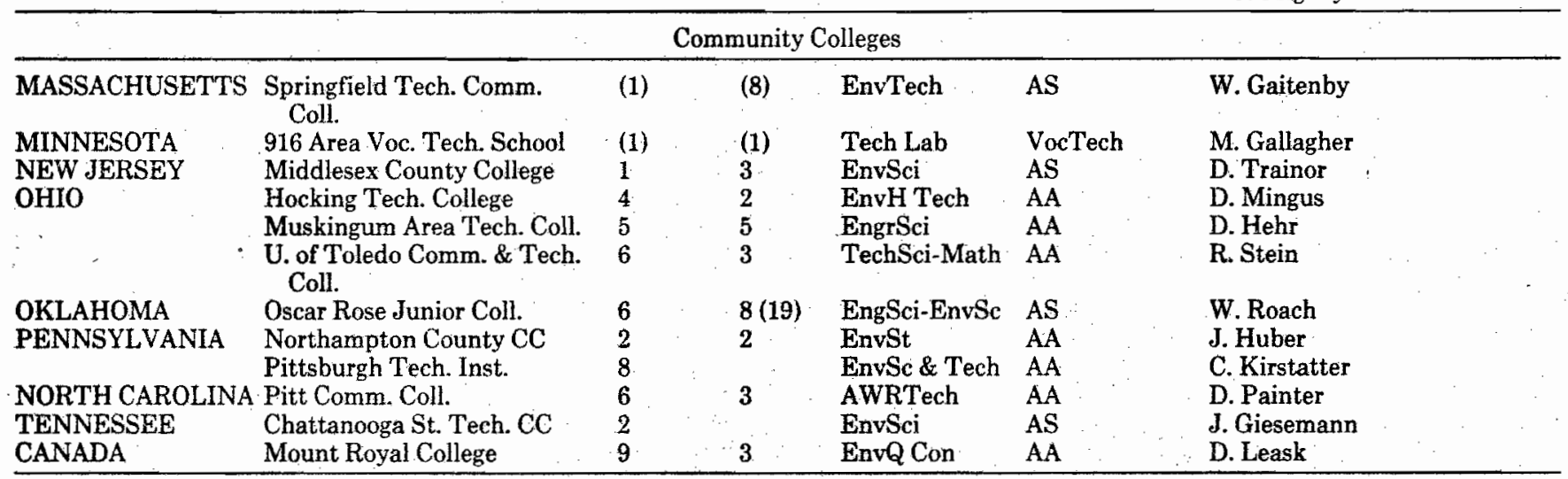

auantities in parentheses reflect undergraduate courses; other quantities reflect graduate courses.

Abbreviations Used

\begin{tabular}{|c|c|c|c|}
\hline $\begin{array}{l}\text { A \& S } \\
\text { Air Cons } \\
\text { Air Env St } \\
\text { Air R } \\
\text { An \& D Sc } \\
\text { Ap H } \\
\text { Ap Sci } \\
\text { Atm Sci } \\
\text { AWR Tech } \\
\text { CE } \\
\text { ChE } \\
\text { ChE Sci } \\
\text { Chem } \\
\text { DSc } \\
\text { Engr } \\
\text { EnvE } \\
\text { EnvH Tech } \\
\text { EnvInd H } \\
\text { EnvSci Tech } \\
\text { EnvE Sci } \\
\text { EnvH } \\
\text { EnvH Sci } \\
\text { Env Mag } \\
\text { Env Oc }\end{array}$ & $\begin{array}{l}\text { Arts and Sciences } \\
\text { Air Conservation } \\
\text { Air Environmental Studies } \\
\text { Air Resources } \\
\text { Animal and Dairy Science } \\
\text { Applied Health } \\
\text { Applied Science } \\
\text { Atmospheric Science } \\
\text { Air and Water Resource Technology } \\
\text { Civil Engineering } \\
\text { Chemical Engineering } \\
\text { Chemical Engineering Science } \\
\text { Chemistry } \\
\text { Doctor of Science } \\
\text { Engineering } \\
\text { Environmental Engineering } \\
\text { Environmental Health Technology } \\
\text { Environmental \& Industrial Health } \\
\text { Environmental Science \& Technology } \\
\text { Environmental Engineering \& Sciences } \\
\text { Environmental Health } \\
\text { Environmental Health Science } \\
\text { Environmental Management } \\
\text { Environmental Occupations }\end{array}$ & $\begin{array}{l}\text { EnvQ Con } \\
\text { EnvR E } \\
\text { EnvS } \\
\text { Env Sci } \\
\text { EnvSE } \\
\text { EnvSt } \\
\text { EnvTech } \\
\text { Erth Sci } \\
\text { H Sci } \\
\text { Hort Sci } \\
\text { ME } \\
\text { Med } \\
\text { Met } \\
\text { OcEnv H } \\
\text { PH } \\
\text { PS } \\
\text { Sci } \\
\text { ThE } \\
\text { Uhn E } \\
\text { WRE } \\
\text { WRM } \\
\text { ThFS } \\
\text { Ubn-EnvE }\end{array}$ & $\begin{array}{l}\text { Environmental Quality Control } \\
\text { Environmental Resources Engineering } \\
\text { Environmental Service } \\
\text { Environmental Science } \\
\text { Environmental Systems Engineering } \\
\text { Environmental Studies } \\
\text { Environmental Technology } \\
\text { Earth Science } \\
\text { Health Science } \\
\text { Horticultural Science } \\
\text { Mechanical Engineering } \\
\text { Medicine } \\
\text { Meteorology } \\
\text { Occupational \& Environmental Health } \\
\text { Public Health } \\
\text { Public Service } \\
\text { Science } \\
\text { Thermal Engineering } \\
\text { Urban Engineering } \\
\text { Water Resources Engineering } \\
\text { Water Resources Management } \\
\text { Thermal Fluid Systems } \\
\text { Urban and Environmental Engineering }\end{array}$ \\
\hline
\end{tabular}

